\title{
THE PREDIMED TRIAL, MEDITERRANEAN DIET AND HEALTH OUTCOMES: HOW STRONG IS THE EVIDENCE?
}

Guasch-Ferré $\mathrm{M}^{\mathrm{a}, \mathrm{b}, \mathrm{c}}$, Salas-Salvadó $\mathrm{J}^{\mathrm{b}, \mathrm{c}}$, Ros $\mathrm{E}^{\mathrm{c}, \mathrm{d}}$, Estruch $\mathrm{R}^{\mathrm{c}, \mathrm{e}}$, Corella $\mathrm{D}^{\mathrm{c}, \mathrm{f}}$, Fitó $\mathrm{M}^{\mathrm{c}, \mathrm{g}}$ and Martínez-González MA ${ }^{\mathrm{a}, \mathrm{c}, \mathrm{g}, \mathrm{h}}$, for the PREDIMED Investigators ${ }^{*}$.

${ }^{a}$ Department of Nutrition, Harvard T.H. Chan School of Public Health, Boston, MA, USA

${ }^{\mathrm{b}}$ Human Nutrition Unit, University Hospital of Sant Joan de Reus, Department of Biochemistry and Biotechnology, Faculty of Medicine and Health Sciences, IISPV, Rovira i Virgili University, Reus, Spain.

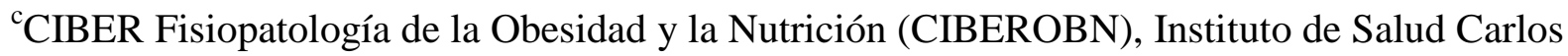
III, Madrid, Spain.

${ }^{\mathrm{d}}$ Lipid Clinic, Endocrinology and Nutrition Service, Institut d'Investigacions Biomèdiques August Pi Sunyer (DIBAPS), Hospital Clinic, University of Barcelona, Barcelona, Spain.

${ }^{\mathrm{e}}$ Department of Internal Medicine, IDIBAPS, Hospital Clinic, University of Barcelona, Barcelona, Spain.

${ }^{\mathrm{f}}$ Department of Preventive Medicine and Public Health, University of Valencia, Valencia, Spain. ${ }^{g}$ Cardiovascular Risk and Nutrition (Regicor Study Group), Hospital del Mar Medical Research Institute (IMIM), Barcelona Spain.

${ }^{h}$ University of Navarra, Department of Preventive Medicine and Public Health, Pamplona, Spain

iIDISNA (Instituto de Investigación Sanitaria de Navarra)

*Arós F, Gómez-Gracia E, Fiol M, Lapetra J, Lamuela-Raventos RM, Tur J, Martinez JA, SerraMajem L and Pintó X. 
*Affilitations:

Department of Cardiology, University Hospital Araba, Vitoria, Spain (FA)

Department of Preventive Medicine, University of Malaga, Malaga, Spain (EG-G)

Institute of Health Sciences, University of Balearic Islands and Son Espases Hospital, Palma de

Mallorca, Spain (MF)

Department of Family Medicine. Research Unit. Primary Care Division of Sevilla, Sevilla, Spain $(\mathrm{JL})$

Nutrition and Food Science Department, XaRTA, INSA, Pharmacy School, University of Barcelona, Barcelona, Spain (RLR)

Research Group on Community Nutrition and Oxidative Stress, University of the Balearic Islands, Palma de Mallorca, Spain (JT)

Department of Nutrition and Food Sciences, University of Navarra, Pamplona, Spain (JAM)

Department of Clinical Sciences, University of Las Palmas de Gran Canaria, Las Palmas, Spain $(\mathrm{LS}-\mathrm{M})$

Lipid Unit, Department of Internal Medicine, IDIBELL-Hospital Universitari de Bellvitge, L'Hospitalet de Llobregat, FIPEC, Barcelona, Spain (XP)

Corresponding author/request for reprints: Miguel A Martínez-González, MD, PhD.

Department of Preventive Medicine and Public Health, University of Navarra, Pamplona, Spain.

Telephone number: +34 948425600; Fax number: +34 948425649; e-mail address:

mamartinez@unav.es. 
Abbreviations: CI, confidence interval; CVD, cardiovascular disease; EVOO, extra-virgin olive oil; HR, hazard ratio; MedDiet, Mediterranean diet; PREDIMED, Prevención con dieta Mediterránea.

Key words: PREDIMED, Mediterranean diet, Cardiovascular disease, clinical trial.

Word count (abstract): 278

Word count text (excludes abstract and references): 3128 


\section{ABSTRACT}

AIMS: We address potential controversies on the health benefits of the MedDiet after PREDIMED, a randomized trial of Mediterranean diet (MedDiet) for primary cardiovascular prevention. We have focused on: a) the PREDIMED study design, b) analysis of PREDIMED data and c) interpretation of its results.

DATA SYNTHESIS: Regarding the design of the trial, its early termination and between-group differences in the intensity of the intervention are potential causes of concern. The planned duration was 6 years but the trial was prematurely stopped when an interim analysis at 4.8-year provided sufficient evidence of benefit for the two MedDiets. In the MedDiet groups supplemented with extra-virgin olive oil or mixed-nuts, the primary composite end point (myocardial infarction, stroke, or cardiovascular death) was reduced by $30 \%$ and $28 \%$ respectively, as compared with the control group. Final results did not change after taking into account the different intensity of educational efforts during the trial. Other potential doubts related to data analysis (e.g., intention to treat versus a per-protocol approach, and consequences of drop-outs) should not be causes of concern. Finally, we addressed alternative interpretations of the effect on all-cause mortality. The protocol-defined primary endpoint was a composite cardiovascular endpoint, not all-cause mortality. To analyze total mortality, we would have needed a much larger sample size and longer follow-up. Therefore, the PREDIMED results cannot be used to draw firm conclusions on MedDiets and all-cause mortality.

CONCLUSIONS: The PREDIMED study was designed to overcome three major problems of previous nutritional research: a) residual confounding, addressed by using a randomized design; b) single-nutrient approaches, by randomizing an overall dietary pattern; and c) the limitations of assessing only intermediate risk markers, by using hard clinical end-points. 


\section{Summary of the PREDIMED trial design and findings}

The "Prevención con Dieta Mediterránea" (PREDIMED) Study is considered to be a pioneer large nutritional trial that focused on an overall dietary pattern for the primary prevention of cardiovascular disease [1]. After the publication of the main results, a few investigators have voiced their concerns on some aspects of the design and results of PREDIMED [2-5] which could comprise the strength of the evidence to recommend a Mediterranean Diet (MedDiet) in cardiovascular prevention. We address these controversies and attempt to provide a thoughtful discussion of each one of them.

PREDIMED was designed to assess the long-term effects of a MedDiet on the primary prevention of cardiovascular disease (CVD) in individuals at high cardiovascular risk (www.predimed.es), aged 55-75 years (men) or 60-80 years (women) [6,7]. The pre-specified primary endpoint was a composite of cardiovascular events including non-fatal myocardial infarction, non-fatal stroke and death from cardiovascular causes. The PREDIMED trial randomized 7477 participants into one of three interventions: MedDiet supplemented with extravirgin olive oil (EVOO), MedDiet supplemented with mixed nuts, or control diet (advice to reduce all dietary fats). EVOO and nuts were provided at no cost; to reinforce adherence, participants in the control group received non-food gifts during the intervention $[6,8,9]$.

The main findings of the trial showed that a MedDiet reduced the incidence of major cardiovascular events by approximately 30\% [Hazard Ratio (HR):0.70(95\% confidence interval, $0.54,0.92)$ and $28 \%[\mathrm{HR}=0.72(0.54,0.96)]$ when supplemented with EVOO or nuts, respectively, in comparison to the control diet [1]. The estimates for absolute risk reductions and numbers needed to treat for the primary end-point and for secondary hard clinical endpoints have been reported [10]. Other benefits included effects on metabolic syndrome [11], adiposity [12], 
cognition $[7,13,14]$ and breast cancer [15]. Intermediate outcomes and changes in classical and novel vascular risk factors together with relevant mechanistic findings have been also reported [16]. PREDIMED obtained also significant reductions in type 2 diabetes by $30 \%$ for the combined MedDiet interventions [HR:0.70 (95\% CI:0.54, 0.92)] [17] and also lower risk of diabetes complications such as retinopathy [18]. Beneficial effects were observed for peripheral arterial disease (66\% reduction in the MedDiet plus EVOO and 50\% reduction in the MedDiet plus nuts) [19]; atrial fibrillation, with a 38\% risk reduction in the MedDiet plus EVOO [20]. Moreover, improvements in lipid profiles and inflammatory status, lower blood pressure and slower progression of subclinical atherosclerosis were observed [21-24]. A meta-analysis reported that there was limited evidence suggesting that a MedDiet with no restriction on fat intake may reduce the incidence of CVD, breast cancer and type 2 diabetes but not all-cause mortality [25]. But PREDIMED provided strong evidence to support the benefits of the MedDiet. While other plant-based dietary patterns can also be deemed as healthy [26], such firstlevel evidence as collected for the MedDiet in a randomized trial with low risk of bias is not available for any other dietary pattern.

The focus on overall dietary patterns is considered nowadays superior to assessing single nutrients or foods [27]. PREDIMED was designed to overcome the problems of the singlenutrient approach and the limitations of assessing only intermediate risk markers or using observational designs. [28] Indeed, PREDIMED is a hallmark study because it is among the very few randomized primary prevention trials of overall food patterns using hard clinical end-points [28].

\section{Current controversies}

Current controversies that have arisen after the PREDIMED trial are addressed in Table 1. 


\section{Study design}

Intensity of intervention. PREDIMED participants randomized to the control group were advised to follow a low-fat diet according to prevailing guidelines when the study was designed in 2003 $[6,7]$. In the MedDiet intervention groups, dieticians ran individual and group dietary-training sessions at baseline and quarterly thereafter encouraging the adherence to the MedDiet.

Adherence to the MedDiet was good but adherence to the intended diet in the control group was suboptimal. This might be due in part to the relatively low intensity of the dietary education and personalized counseling implemented for the control group during the first 3 years of the trial (up to October 2006), when only a leaflet describing the low-fat diet was given on a yearly basis. However, after 3 years, the protocol was amended and control group participants received quarterly dietary education with exactly the same frequency and intensity than those in the two active MedDiet groups, which accounted for $60 \%$ of the time they were in the trial. Specific analyses, stratified by recruitment date, splitting the cohort in two (cut-off point: enrollment before or after October 2006) yielded adjusted HR:0.77 (95\% CI: 0.59, 1.00) for participants recruited before October 2006 and HR:0.49 (95\% CI: 0.26, 0.92) for those recruited later (P for interaction=0.21) when comparing the MedDiet (both groups merged) versus control. Only participants in the control group recruited before October 2006 were exposed to the low-level intensity education on low-fat diet; however, the effect was apparently weaker among them. This would only add further support to our hypothesis and allows to dispel any criticism that the beneficial effect of the MedDiet was due to lower educational intensity in the control group. Regarding acceptability of low-fat diets, findings from landmark clinical trials of low-calorie diets with different macronutrient composition for weight loss in overweight/obese participants support the use of moderate fat diets including olive oil and nuts as an alternative to low-fat diets 
because of increased palatability, better compliance, and superior long-term sustainability and effectiveness against cardiometabolic disease [29-31]. In the Women's Health Initiative Dietary Modification Trial [32], postmenopausal women were randomized to usual diet versus a low-fat diet intervention with the goal of reducing fat intake to $20 \%$ of energy; although participants reduced fat intake, it fell short of the target, reaching $29 \%$ of energy after 8.1 years of intervention, underlining the difficulty of participants to lower their fat intake to the theoretical standards of a low-fat diet. Furthermore, the results of that study demonstrated that reducing total fat failed to reduce the risk of incident CVD in contrast with PREDIMED [33]. Finally, PREDIMED results do not contradict that low-fat diets may also be cardio-protective, albeit large clinical trial evidence is lacking.

Early termination of the trial. Benefits of the MedDiets in PREDIMED could have been overestimated because the trial was stopped prematurely on the basis of early significant benefits of the intervention after a median follow-up time of 4.8 years, while the planned duration was 6 years. Trials that terminate before the scheduled closing date tend to have magnified results. However, preplanned interim analyses, stopping rules, and the appointment of a Data and Safety Monitoring Board (DSMB) in the PREDIMED trial ensured transparency and credibility of decision making during the trial, in particular with respect to premature discontinuation [34,35]. In the protocol we defined in anticipation regular interim assessments during the trial and two independent statistical investigators blinded to the treatment allocation carried out the analyses. The decision to stop the trial was made in agreement with the rules of the protocol and the recommendations of the DSMB on the grounds that it was unethical to continue a trial that has already shown that an intervention is superior in terms of CVD event rates, hence it should be offered to all participants. The reduction in CVD risk found in the PREDIMED Study was also 
consistent with the predicted benefits derived from observational data on individual dietary components reported by The Global Burden of Diseases, Nutrition, and Chronic Diseases Expert (NUTRICODE) Group [36].

\section{Analysis of data}

Intention to treat versus protocol analyses. Despite the recommendation to reduce consumption of all types of dietary fat, including olive oil and nuts, the control group did not achieve a sufficiently low fat intake. At baseline, total fat intake $(\% \mathrm{E})$ in the control group was $39.0 \pm 7.0$ and it was reduced to $37.0 \pm 7.0$ at the end of the trial. The intake of saturated fat $(\% \mathrm{E})$ in the control group was reduced from $10.0 \pm 2.3$ to $9.1 \pm 2.1$. Admittedly, these observed reductions were both of small size. However, as planned in the protocol, the main analyses on the PREDIMED trial followed an intention-to-treat approach, which evaluated the advice strategy of the PREDIMED interventions. The dilemma between 'intention- to-treat' versus 'per protocol' approaches is a common problem in dietary trials. Methodologically, it seems better to select the intention-to-treat approach because it completely preserves the advantages of randomization. But it should be acknowledged that the intention-to-treat approach evaluates the advice strategy, whereas the per protocol analysis is more appropriate to assess what participants actually consumed during the trial. The first approach, that the PREDIMED (and all major cardiovascular trials) used, proves the effects of a treatment strategy and the other the effect of consuming foods. Intention-to-treat takes advantage of randomization in order to avoid confounding by both measured and unmeasured potential confounders, the per protocol approach should use multivariable methods to reduce confounding, but can control only for those confounders which have been measured. In any case, the PREDIMED group did report a per 
protocol analysis among diabetics showing that a low-fat diet ( $<30 \%$ of total energy as fat) was not associated with any cardiovascular protection [37].

The benefits observed in the MedDiet groups in PREDIMED might be arguably explained by a higher intake of simple sugars in the control group, as a replacement for high-fat foods, and also by lower consumption of fatty fish. In PREDIMED, control group participants were advised to consume whole-grain cereals and other complex carbohydrates, not refined cereals, sweets or sugar-rich products. The intake of refined cereal and potatoes $(60 \mathrm{~g})$ was reduced by $-0.31(-0.39$, -0.23) (mean (95\% CI) in the control group, and no between-group significant differences were observed with the MedDiet groups. Fatty fish was indeed discouraged in the control group, whose participants reduced mean marine n-3 fatty acid intake from 0.8 to $0.7 \mathrm{~g} / \mathrm{day}$. Yet, this level of intake is still above the recommended intake of $0.5 \mathrm{~g} /$ day for cardiovascular protection [38], meaning that the small decrease in fatty fish consumption by control participants was irrelevant concerning cardiovascular risk.

Differences in baseline characteristics of participants. There were some statistically significant differences in baseline characteristics among the three intervention groups, such as a higher percentage of women (in the control group), and that more individuals had obesity and used diuretics and oral hypoglycemic medication in the control group, even though we used a standard computerized randomization method [1]. However, differences between groups were minor in size and of little clinical relevance. Importantly, these minor imbalances will produce a lower rate of cardiovascular events in the control group and will obscure the true differences, because female sex showed the strongest inverse association with the endpoint and the women proportion was higher in control than in intervention groups. Furthermore, results did not substantially change when these variables were included as cofounders in the statistical models. 
Drop-outs. The number of dropouts was larger in the control that in the MedDiet groups. This differential attrition (rendering different denominators of person-years for the rates in the three groups) might have contributed to a greater benefit of the MedDiet supplemented with extravirgin olive oil (where the count of events was not substantially lower, but the person-years denominator was larger). To include only the observed times of follow-up for each participant is the most appropriate and correct approach in any analysis for time-to-event (survival analyses). Therefore, the main results of PREDIMED were free from selection bias because we did not include the missing times of individuals who dropped out after they left the trial, i.e. we censored them. Sensitivity analyses including imputation of data for missing values and of participants who dropped out were also conducted and they were consistent with the primary analysis. Additionally, dropouts had a worse baseline cardiovascular risk profile, which suggests a bias toward benefit in the control group were dropouts were higher; namely, without dropouts, there could have been even higher cardiovascular risk status and more CVD events in the control group than in the MedDiet groups.

\section{Interpretation of results}

Questions on body weight and adiposity. A recurrent question is whether the high-fat MedDiet might promote increased body weight. High-fat foods such as the generous amount of olive oil and nuts consumed by participants in the PREDIMED trial can be thought as leading to weight gain and promote obesity. Despite this popular belief, available scientific evidence suggests that frequent consumption of olive oil [39] or nuts [40] in the context of a healthy diet does not lead to any appreciable weight gain. Obesity is essentially an imbalance between energy intake and expenditure and there is no conclusive human evidence that, controlling for energy intake, any particular energy-generating nutrient has substantial inherent obesity-promoting consequences. 
We have also specifically shown in the PREDIMED that the high-vegetable fat MedDiet did not promote adiposity $[12,41]$. After 5 years of intervention, median total fat intake represented $37 \%$ and $42 \%$ of energy in the control and MedDiet groups. But participants in all three groups marginally reduced body weight and increased waist circumference, as expected in older adults. Adjusted differences in 5-year changes in body weight in the MedDiet with olive oil and nuts groups were $-0.43(95 \% \mathrm{CI},-0.86,-0.01)$ and $-0.08(-0.50,+0.35) \mathrm{kg}$, respectively, versus control. Respective differences for the changes in waist circumference were $-0.55(-1.16,-0.06)$ and 0.94(-1.60, -0.27) cm [12]. Thus, a long-term intervention with an unrestricted-calorie, high-fat, high-vegetable fat MedDiet was associated with decreases in body weight and less gain in central adiposity. This apparent paradox might be explained by the fact that consumption of these foods has been shown to increase satiety, induce thermogenesis, increase resting energy expenditure and post-prandial fat oxidation, and decrease energy intake from other sources by food compensation $[40,42,43]$. The quality of dietary fat, especially in diets that favor plant-based fats over animal fats, has a more important role in determining the risk of chronic diseases such as type 2 diabetes than the total amount of fat in the diet $[25,28,29,41,44-46]$. According to PREDIMED, these salutary effects are likely to occur without undue changes in body weight or adiposity.

Attribution of observed benefits to supplemental foods or to the food pattern. Controversy persists on whether the benefits should be attributed to the supplemental foods (extra-virgin olive oil and mixed nuts), or rather to the overall MedDiet pattern. In addition, a third MedDiet group educated to follow the MedDiet but not receiving supplements may had enabled further discrimination in effects between intervention groups and the control group. Unfortunately, that additional group was not included in the design of the PREDIMED trial, the 2 groups receiving 
education to follow the MedDiet also received supplements (EVOO and nuts). Therefore, a direct comparison is not possible and it is difficult to disentangle the effect of these two salutary foods from that of the whole food pattern. However, we believe that synergy among all healthy components of the diet was instrumental in obtaining the observed risk reduction [47]. Dietary patterns often tell us more on associations with disease risk than do single foods and/or nutrients [26]. Notwithstanding, olive oil and nuts provide large quantities of vegetable fats rich in monounsaturated fatty acids and some nuts (e.g., walnuts) also contain sizable amounts of polyunsaturated fatty acids, which have been associated with many health benefits $[25,44-$ 46,48-54]. In PREDIMED, a 14-item screener of adherence to the MedDiet was developed to both assess and improve compliance at quarterly visits of the participants with dietitians [55]. Participants in the MedDiet groups significantly improved most of the individual score components with significant differences versus control in 12 out of the 14 items [1]. Therefore, small changes were attained not only in nuts or olive oil, but in the overall food pattern. Each 2point increment in baseline adherence to the 14 -item score was associated with a $25 \%$ reduction in CVD [54]. This illustrates the greater potential of the whole dietary pattern. In relation to this point, another question that has been often raised is that, for some of the analyzed outcomes, the MedDiet plus EVOO was more beneficial than the MedDiet plus nuts, or otherwise. However, the PREDIMED trial was not designed to compare the effect between both intervention groups and in most analyses the 95\% CIs overlapped between the two MedDiet groups. Hence, there is no reason to believe that one of the MedDiet interventions was more effective than the other.

Concerns regarding specific CVD outcomes. It has been suggested that the MedDiet only protected from stroke, but not from myocardial infarction or overall CVD. In the PREDIMED protocol we pre-defined a composite of cardiovascular events, as customary in most 
pharmacologic CVD prevention trials, and we showed that the MedDiet interventions were associated with a significant reduction in this pre-defined primary outcome of composite of CVD events compared to the control diet $[1,6,10]$. Among components of the primary outcome, we observed significantly reduced rates of stroke for both MedDiets, but only a trend for reduced myocardial infarction. The explanation is simple, as more strokes than myocardial infarction or CVD deaths events occurred, hence the statistical power of the study favored the detection of differences in stroke. The trial was neither designed nor powered to individually assess each of the individual components of the primary end-point. In addition, the issues of competing risks are better tackled by defining a composite end-point according to the state-of-the-art methodology [56].

Effects on all-cause mortality. A lower death rate was observed in the two intervention groups merged together (10.5 deaths per 1000 persons-years) than in the control group (11.7 deaths per 1000 persons-years). However, this difference did not reach the conventional threshold $(\mathrm{p}=0.05)$ of statistical significance. Because in PREDIMED we found no significant between-group differences in total mortality [HR:0.89 (95\% CI, 0.71, 1.12) for MedDiet versus control], it was argued that the only effect of the intervention was to replace CVD mortality by other causes of death. However, most likely the number of deaths prevented with the nutritional interventions may not be large enough to show statistically significant differences between groups in all-cause mortality. In other words, our study was not sufficiently powered to detect differences in allcause mortality.

Too early to be true. Lastly, it has also been suggested that the separation of the Kaplan-Meir curves of CVD events along time by treatment allocation occurred too early and that this was implausible. Yet, this early protective effect of the intervention with MedDiets might be 
explained by prompt reductions in blood pressure, LDL cholesterol and inflammation, as well as an increase in HDL cholesterol and insulin sensitivity. In fact, our pilot study showed that Mediterranean diets conferred a substantial benefit with regard to classical and emergent cardiovascular risk factors after only 3 months since randomization [57,58]. Moreover, we recognize that 5 years is a long period to adhere to a dietary intervention.

In conclusion, most research on dietary patterns has been conducted in the context of observational studies or short-term trials with not hard end-points. The PREDIMED trial has demonstrated with strong evidence and low risk of bias that the traditional MedDiet provides protection against CVD in individuals at high cardiovascular risk. The methodology and results of PREDIMED represent important steps forward for designing dietary studies. Future dietary studies should learn from those aspects of PREDIMED specifically designed to overcome three major common problems of nutritional research: a) the problem of residual confounding in observational studies; b) the problem of single-nutrient approaches; and c) the limitations of assessing only intermediate risk markers instead of using hard clinical end-points as outcomes. Importantly, PREDIMED findings support that overall dietary patterns rich in high-unsaturated fat foods from natural vegetable sources are more beneficial for CVD prevention than a lower fat diet without incurring undue weight gain. Our findings also reinforce the need to preserve and promote the traditional MedDiet as a valuable cultural heritage. We have contributed to investigate these associations in a Mediterranean population at high cardiovascular risk. Public health policies and appropriate nutritional recommendations to promote the MedDiet together with a healthy lifestyle should be a cornerstone for the prevention of chronic disease at a national and international level. 


\section{Acknowlegements}

The authors are especially thankful to Dr. José C Florez (Massachusetts General Hospital, Boston, USA), for his insightful comments, suggestions and ideas during the conception of this article.

\section{Declarations of interest}

Supported by the official funding agency for biomedical research of the Spanish government, Instituto de Salud Carlos III (ISCIII), through grants provided to research networks specifically developed for the trial (RTIC G03/140, to Dr. Estruch; RTIC RD 06/0045, to Dr. Martínez González and through Centro de Investigación Biomédica en Red de Fisiopatología de la Obesidad y Nutrición [CIBERobn]), and by grants from Centro Nacional de Investigaciones Cardiovasculares (CNIC 06/2007), Fondo de Investigación Sanitaria-Fondo Europeo de Desarrollo Regional (PI04-2239, PI 05/2584, CP06/00100, PI07/0240, PI07/1138, PI07/0954, PI 07/0473, PI10/01407, PI10/02658, PI11/01647, and P11/02505), Ministerio de Ciencia e Innovación (AGL-2009-13906-C02 and AGL2010-22319-C03), Fundación Mapfre 2010, Consejería de Salud de la Junta de Andalucía (PI0105/2007), Public Health Division of the Department of Health of the Autonomous Government of Catalonia, Generalitat Valenciana (ACOMP06109, GVACOMP2010-181, GVACOMP2011-151, CS2010-AP-111, and CS2011 AP-042), and Regional Government of Navarra (P27/2011). Dr. Estruch reports serving on the board of and receiving lecture fees from the Research Foundation on Wine and Nutrition (FIVIN); serving on the boards of the Beer and Health Foundation and the European Foundation for Alcohol Research (ERAB); receiving lecture fees from Cerveceros de España and Sanofi Aventis; and receiving grant support through his institution from Novartis. Dr. Ros reports 
serving on the board of and receiving travel support, as well as grant support through his institution, from the California Walnut Commission; serving on the board of the Flora Foundation (Unilever); serving on the board of and receiving lecture fees from Roche; serving on the board of and receiving grant support through his institution from Amgen; receiving consulting fees from Damm and Abbott Laboratories; receiving consulting fees and lecture fees, as well as grant support through his institution, from Merck; receiving lecture fees from Danone, Pace, AstraZeneca, and Rottapharm; receiving lecture fees and payment for the development of educational presentations, as well as grant support through his institution, from Ferrer; receiving payment for the development of educational presentations from Recordati; and receiving grant support through his institution from Sanofi-Aventis, Takeda, Daiichi Sankyo, Nutrexpa, Feiraco, Unilever, and Karo Bio. Dr.Salas-Salvadó reports serving on the board of and receiving grant support through his institution from the International Nut and Dried Fruit Council; receiving consulting fees from Danone; and receiving grant support through his institution from Eroski and Nestlé. No other potential conflict of interest relevant to this article was reported. 


\section{REFERENCES}

[1] Estruch R, Ros E, Salas-Salvado J, Covas MI, Corella D, Aros F, et al. Primary prevention of cardiovascular disease with a Mediterranean diet. N Engl J Med 2013;368:1279-90. doi:10.1056/NEJMoa1200303.

[2] Kopel E, Sidi Y, Kivity S. Mediterranean diet for primary prevention of cardiovascular disease. N Engl J Med 2013;369:672. doi:10.1056/NEJMc1306659\#SA1.

[3] Ornish D. Mediterranean diet for primary prevention of cardiovascular disease. N Engl J Med 2013;369:675-6. doi:10.1056/NEJMc1306659\#SA6.

[4] Kopel E, Sidi Y, Kivity S. Prevention of diabetes with Mediterranean diets. Ann Intern Med 2014;161:157. doi:10.7326/L14-5014.

[5] Ioannidis JPA. Implausible results in human nutrition research. BMJ 2013;347:f6698.

[6] Martinez-Gonzalez MA, Corella D, Salas-Salvado J, Ros E, Covas MI, Fiol M, et al. Cohort profile: design and methods of the PREDIMED study. Int J Epidemiol 2012;41:377-85. doi:10.1093/ije/dyq250.

[7] Martínez-Lapiscina EH, Clavero P, Toledo E, San Julián B, Sanchez-Tainta A, Corella D, et al. Virgin olive oil supplementation and long-term cognition: the PREDIMED-

NAVARRA randomized, trial. J Nutr Health Aging 2013;17:544-52. doi:10.1007/s12603013-0027-6.

[8] Zazpe I, Sanchez-Tainta A, Estruch R, Lamuela-Raventos RM, Schröder H, Salas-Salvado $\mathrm{J}$, et al. A large randomized individual and group intervention conducted by registered dietitians increased adherence to Mediterranean-type diets: the PREDIMED study. J Am Diet Assoc 2008; 108:1134-44.

[9] Downer MK, Gea A, Stampfer M, Sánchez-Tainta A, Corella D, Salas-Salvadó J, et al. 
Predictors of short- and long-term adherence with a Mediterranean-type diet intervention: the PREDIMED randomized trial. Int J Behav Nutr Phys Act 2016;13:67. doi:10.1186/s12966-016-0394-6.

[10] Martínez-González MA, Salas-Salvadó J, Estruch R, Corella D D, Fitó M, Ros E. Benefits of the Mediterranean Diet: Insights from the PREDIMED Study. Prog Cardiovasc Dis 2015;58:50-60. doi:10.1016/j.pcad.2015.04.003.

[11] Babio N, Toledo E, Estruch R, Ros E, Martínez-González MA, Castañer O, et al. Mediterranean diets and metabolic syndrome status in the PREDIMED randomized trial. CMAJ 2014;186:E649-57. doi:10.1503/cmaj.140764.

[12] Estruch R, Martínez-González MA, Corella D, Salas-Salvadó J, Fitó M, Chiva-Blanch G, et al. Effect of a high-fat Mediterranean diet on bodyweight and waist circumference: a prespecified secondary outcomes analysis of the PREDIMED randomised controlled trial. Lancet Diabetes Endocrinol 2016. doi:10.1016/S2213-8587(16)30085-7.

[13] Martínez-Lapiscina EH, Clavero P, Toledo E, Estruch R, Salas-Salvadó J, San Julián B, et al. Mediterranean diet improves cognition: the PREDIMED-NAVARRA randomised trial. J Neurol Neurosurg Psychiatry 2013;84:1318-25. doi:10.1136/jnnp-2012-304792.

[14] Valls-Pedret C, Sala-Vila A, Serra-Mir M, Corella D, de la Torre R, Martínez-González MÁ, et al. Mediterranean Diet and Age-Related Cognitive Decline: A Randomized Clinical Trial. JAMA Intern Med 2015;175:1094-103. doi:10.1001/jamainternmed.2015.1668.

[15] Toledo E, Salas-Salvadó J, Donat-Vargas C, Buil-Cosiales P, Estruch R, Ros E, et al. Mediterranean Diet and Invasive Breast Cancer Risk Among Women at High Cardiovascular Risk in the PREDIMED Trial: A Randomized Clinical Trial. JAMA Intern 
Med 2015;175:1752-60. doi:10.1001/jamainternmed.2015.4838.

[16] Ros E, Martínez-González MA, Estruch R, Salas-Salvadó J, Fitó M, Martínez JA, et al. Mediterranean diet and cardiovascular health: Teachings of the PREDIMED study. Adv Nutr 2014;5:330S-6S. doi:10.3945/an.113.005389.

[17] Salas-Salvadó J, Bulló M, Estruch R, Ros E, Covas M-I, Ibarrola-Jurado N, et al. Prevention of diabetes with Mediterranean diets: a subgroup analysis of a randomized trial. Ann Intern Med 2014;160:1-10. doi:10.7326/M13-1725.

[18] Díaz-López A, Babio N, Martínez-González MA, Corella D, Amor AJ, Fitó M, et al. Mediterranean Diet, Retinopathy, Nephropathy, and Microvascular Diabetes Complications: A Post Hoc Analysis of a Randomized Trial. Diabetes Care 2015;38:2134-41. doi:10.2337/dc15-1117.

[19] Ruiz-Canela M, Estruch R, Corella D, Salas-Salvadó J, Martínez-González MA. Association of Mediterranean diet with peripheral artery disease: the PREDIMED randomized trial. JAMA 311:415-7. doi:10.1001/jama.2013.280618.

[20] Martínez-González MÁ, Toledo E, Arós F, Fiol M, Corella D, Salas-Salvadó J, et al. Extravirgin olive oil consumption reduces risk of atrial fibrillation: the PREDIMED (Prevención con Dieta Mediterránea) trial. Circulation 2014;130:18-26. doi:10.1161/CIRCULATIONAHA.113.006921.

[21] Murie-Fernandez M, Irimia P, Toledo E, Martínez-Vila E, Buil-Cosiales P, SerranoMartínez M, et al. Carotid intima-media thickness changes with Mediterranean diet: a randomized trial (PREDIMED-Navarra). Atherosclerosis 2011;219:158-62. doi:10.1016/j.atherosclerosis.2011.06.050.

[22] Toledo E, Hu FB, Estruch R, Buil-Cosiales P, Corella D, Salas-Salvadó J, et al. Effect of 
the Mediterranean diet on blood pressure in the PREDIMED trial: results from a randomized controlled trial. BMC Med 2013;11:207. doi:10.1186/1741-7015-11-207.

[23] Doménech M, Roman P, Lapetra J, García de la Corte FJ, Sala-Vila A, de la Torre R, et al. Mediterranean diet reduces 24-hour ambulatory blood pressure, blood glucose, and lipids: one-year randomized, clinical trial. Hypertension 2014;64:69-76.

doi:10.1161/HYPERTENSIONAHA.113.03353.

[24] Sala-Vila A, Romero-Mamani E-S, Gilabert R, Núñez I, de la Torre R, Corella D, et al. Changes in ultrasound-assessed carotid intima-media thickness and plaque with a Mediterranean diet: a substudy of the PREDIMED trial. Arterioscler Thromb Vasc Biol 2014;34:439-45. doi:10.1161/ATVBAHA.113.302327.

[25] Bloomfield HE, Koeller E, Greer N, MacDonald R, Kane R, Wilt TJ. Effects on Health Outcomes of a Mediterranean Diet With No Restriction on Fat Intake: A Systematic Review and Meta-analysis. Ann Intern Med 2016. doi:10.7326/M16-0361.

[26] Schwingshackl, L HG. Diet quality as assessed by the Healthy Eating Index, the Alternate Healthy Eating Index, the Dietary Approaches to Stop Hypertension score, and health outcomes: a systematic review and meta-analysis of cohort studies. J Acad Nutr Diet 2015;115:780-800.

[27] Hu FB. Dietary pattern analysis: a new direction in nutritional epidemiology. Curr Opin Lipidol 2002;13:3-9.

[28] Satija A, Yu E, Willett WC, Hu FB. Understanding nutritional epidemiology and its role in policy. Adv Nutr 2015;6:5-18. doi:10.3945/an.114.007492.

[29] Tobias DK, Chen M, Manson JE, Ludwig DS, Willett W HF. Effect of low-fat diet interventions versus other diet interventions on long-term weight change in adults: a 
systematic review and meta-analysis. Lancet Diabetes Endocrinol 2015;3:968-79.

[30] Kastorini C-M, Milionis HJ, Esposito K, Giugliano D, Goudevenos JA, Panagiotakos DB. The effect of Mediterranean diet on metabolic syndrome and its components: a metaanalysis of 50 studies and 534,906 individuals. J Am Coll Cardiol 2011;57:1299-313. doi:10.1016/j.jacc.2010.09.073.

[31] Li Y, Hruby A, Bernstein AM, Ley SH, Wang DD, Chiuve SE, et al. Saturated Fats Compared With Unsaturated Fats and Sources of Carbohydrates in Relation to Risk of Coronary Heart Disease. J Am Coll Cardiol 2015;66:1538-48. doi:10.1016/j.jacc.2015.07.055.

[32] Howard B V, Van Horn L, Hsia J, Manson JE, Stefanick ML, Wassertheil-Smoller S, et al. Low-fat dietary pattern and risk of cardiovascular disease: the Women's Health Initiative Randomized Controlled Dietary Modification Trial. JAMA 2006;295:655-66. doi:10.1001/jama.295.6.655.

[33] Mozaffarian D. Dietary and Policy Priorities for Cardiovascular Disease, Diabetes, and Obesity: A Comprehensive Review. Circulation 2016;133:187-225. doi:

10.1161/CIRCULATIONAHA.115.018585.

[34] Bassler D, Montori VM, Briel M, Glasziou P, Walter SD, Ramsay T, et al. Reflections on meta-analyses involving trials stopped early for benefit: is there a problem and if so, what is it? Stat Methods Med Res 2013;22:159-68. doi:10.1177/0962280211432211.

[35] Tyson JE, Pedroza C, Wallace D, D’Angio C, Bell EF, Das A. Stopping guidelines for an effectiveness trial: what should the protocol specify? Trials 2016;17:240. doi:10.1186/s13063-016-1367-4.

[36] Mozaffarian D. Mediterranean diet for primary prevention of cardiovascular disease. N 
Engl J Med 2013;369:673-4. doi:10.1056/NEJMc1306659\#SA3.

[37] Martínez-González MA, Salas-Salvadó J, Estruch R. Intensive lifestyle intervention in type 2 diabetes. N Engl J Med 2013;369:2357. doi:10.1056/NEJMc1312802\#SA4.

[38] ISSFAL, Intake of PUFA in Healthy Adults, http://www.issfal.org/statements/pufarecommendations/statement-3. Accessed October 20, 2016.

[39] Bes-Rastrollo M, Sánchez-Villegas A, de la Fuente C, de Irala J, Martinez JA, MartínezGonzález MA. Olive oil consumption and weight change: the SUN prospective cohort study. Lipids 2006;41:249-56.

[40] Flores-Mateo G, Rojas-Rueda D, Basora J, Ros E, Salas-Salvado J. Nut intake and adiposity: meta-analysis of clinical trials. Am J Clin Nutr 2013;97:1346-55. doi:10.3945/ajen.111.031484; 10.3945/ajen.111.031484.

[41] Mozaffarian D. Food and weight gain: time to end our fear of fat. Lancet Diabetes Endocrinol 2016;4:633-5. doi: 10.1016/S2213-8587(16)30106-1.

[42] Natoli S, McCoy P. A review of the evidence: nuts and body weight. Asia Pac J Clin Nutr 2007;16:588-97.

[43] Rajaram S, Sabate J. Nuts, body weight and insulin resistance. Br J Nutr 2006;96 Suppl 2:S79-86.

[44] Perez-Jimenez F, Alvarez de Cienfuegos G, Badimon L, Barja G, Battino M, Blanco A, et al. International conference on the healthy effect of virgin olive oil. Eur J Clin Invest 2005;35:421-4. doi:10.1111/j.1365-2362.2005.01516.x.

[45] Trichopoulou A, Martínez-González MA, Tong TY, Forouhi NG, Khandelwal S, Prabhakaran D, et al. Definitions and potential health benefits of the Mediterranean diet: views from experts around the world. BMC Med 2014;12:112. doi:10.1186/1741-7015- 
12-112.

[46] Martínez-González MA, Dominguez LJ, Delgado-Rodríguez M, Martínez-González, Miguel Ángel; Domínguez, Ligia J; Delgado-Rodríguez M. Olive oil consumption and risk of CHD and/or stroke: a meta-analysis of case-control, cohort and intervention studies. Br J Nutr. 2014 Jul 28;112(2):248-59. doi: 10.1017/S0007114514000713.

[47] Jacobs DR, Tapsell LC. Food synergy: the key to a healthy diet. Proc Nutr Soc 2013;72:200-6. doi:10.1017/S0029665112003011.

[48] Ros E. Nuts and CVD. Br J Nutr 2015;113 Suppl. doi:10.1017/S0007114514003924.

[49] Ruano J1, Lopez-Miranda J, Fuentes F, Moreno JA, Bellido C, Perez-Martinez P, Lozano A, Gómez P, Jiménez Y PJF. Phenolic content of virgin olive oil improves ischemic reactive hyperemia in hypercholesterolemic patients. J Am Coll Cardiol 2005;46:1864-8.

[50] Guasch-Ferré M, Hu FB, Martínez-González MA, Fitó M, Bulló M, Estruch R, et al. Olive oil intake and risk of cardiovascular disease and mortality in the PREDIMED Study. BMC Med 2014;12:78. doi:10.1186/1741-7015-12-78.

[51] Guasch-Ferre M, Bullo M, Martinez-Gonzalez MA, Ros E, Corella D, Estruch R, et al. Frequency of nut consumption and mortality risk in the PREDIMED nutrition intervention trial . BMC Med 2013;11:164. doi:10.1186/1741-7015-11-164; doi:10.1186/1741-701511-164.

[52] Schwingshackl L, Hoffmann G. Monounsaturated fatty acids, olive oil and health status: a systematic review and meta-analysis of cohort studies. Lipids Health Dis 2014;13:154. doi:10.1186/1476-511X-13-154.

[53] Schwingshackl, L. Hoffman G. Mediterranean dietary pattern, inflammation and endothelial function: a systematic review and meta-analysis of intervention trials. Nutr 
Metab Cardiovasc Dis 2014;24:929-39.

[54] Schröder H, Salas-Salvadó J, Martínez-González MA, Fíto M, Corella D, Estruch R, et al. Baseline adherence to the Mediterranean diet and major cardiovascular events: Prevención con Dieta Mediterránea trial. JAMA Intern Med 2014;174:1690-2.

doi:10.1001/jamainternmed.2014.3463.

[55] Schröder H, Fitó M, Estruch R, Martínez-González MA, Corella D, Salas-Salvadó J, et al. A short screener is valid for assessing Mediterranean diet adherence among older Spanish men and women. J Nutr 2011;141:1140-5. doi:10.3945/jn.110.135566.

[56] Andersen PK, Geskus RB, de Witte T, Putter H. Competing risks in epidemiology: possibilities and pitfalls. Int J Epidemiol 2012;41:861-70. doi:10.1093/ije/dyr213.

[57] Estruch R, Martínez-González MA, Corella D, Salas-Salvadó J, Ruiz-Gutiérrez V, Covas MI, et al. Effects of a Mediterranean-style diet on cardiovascular risk factors: a randomized trial. Ann Intern Med 2006;145:1-11. doi:145/1/1 [pii].

[58] Fito M, Guxens M, Corella D, Saez G, Estruch R, de la Torre R, et al. Effect of a traditional Mediterranean diet on lipoprotein oxidation: a randomized controlled trial . Arch Intern Med 2007;167:1195-203. doi:10.1001/archinte.167.11.1195. 
TABLE 1. Summary table: Current controversies about the PREDIMED trial.

\begin{tabular}{|c|c|}
\hline Frequent questions and/or criticisms & Explanations \\
\hline \multicolumn{2}{|l|}{ Study design } \\
\hline $\begin{array}{l}\text { Intensity of intervention. Participants in the } \\
\text { control group did not achieve a low-fat intake }\end{array}$ & $\begin{array}{l}\text {-Participants were older adults belonging to a } \\
\text { Mediterranean country in which olive oil is } \\
\text { used abundantly in the kitchen and at the } \\
\text { table. } \\
\text { - Poor palatability of low-fat foods hinders } \\
\text { long-term compliance. } \\
\text { - In Mediterranean countries use of olive oil } \\
\text { permits abundant intake of cooked vegetables } \\
\text { and salads, hence recommending increased } \\
\text { consumption of vegetables while limiting } \\
\text { olive oil made little sense to participants in } \\
\text { the control diet group. }\end{array}$ \\
\hline $\begin{array}{l}\text { Intensity of intervention. The low-fat diet } \\
\text { advice was not as intense as the advice to } \\
\text { follow the MedDiet in the active intervention } \\
\text { groups }\end{array}$ & $\begin{array}{l}\text { - Initially, the control group received a less } \\
\text { intense intervention. This was corrected even } \\
\text { before the recruitment was completed. } \\
\text { - The final results did not change when we } \\
\text { took into account the different intensity of the } \\
\text { educational efforts in the control group in } \\
\text { some participants. On the contrary, the greater } \\
\text { effectiveness of the MedDiet was even } \\
\text { stronger when a stronger intervention to foster } \\
\text { the low-fat diet was applied to the control diet } \\
\text { group. }\end{array}$ \\
\hline $\begin{array}{l}\text { Early termination. The PREDIMED study } \\
\text { was stopped prematurely and this could have } \\
\text { magnified the main results }\end{array}$ & $\begin{array}{l}\text { - The observed benefit was strongly } \\
\text { consistent with predicted benefits calculated } \\
\text { from prior observational data. } \\
\text {-The planned duration of the trial was } 6 \text { years. } \\
\text { It was stopped due to evidence of benefit after } \\
4.8 \text { years of median follow-up. } \\
\text { - Preplanned interim analyses, stopping rules, } \\
\text { and the presence of a Data Safety and } \\
\text { Monitoring Board assured transparency and } \\
\text { credibility in the decision of stopping the trial. } \\
\text { - It would have been unethical to continue the } \\
\text { trial with high-risk participants allocated to }\end{array}$ \\
\hline
\end{tabular}




\begin{tabular}{|c|c|}
\hline & the control group. \\
\hline \multicolumn{2}{|l|}{ Analysis of data } \\
\hline $\begin{array}{l}\text { Intention to treat vs protocol. Higher intake of } \\
\text { sugar and refined carbohydrates by control } \\
\text { group participants may explain the advantages } \\
\text { of the MedDiet }\end{array}$ & $\begin{array}{l}\text { - We recommended whole-grain } \\
\text { carbohydrates instead of sugar or refined } \\
\text { carbohydrates in the control group. } \\
\text {-No significant differences in added sugars, } \\
\text { refined carbohydrates or potatoes were found } \\
\text { at the end of the trial between the three } \\
\text { intervention groups. }\end{array}$ \\
\hline $\begin{array}{l}\text { Intention to treat vs protocol. The limitation } \\
\text { to consume fatty fish in the control group may } \\
\text { explain the advantage of the MedDiet in the } \\
\text { other two groups }\end{array}$ & $\begin{array}{l}\text { - The cardiovascular benefits of fish are } \\
\text { usually observed with } 3 \text { servings/week, with } \\
\text { no additional benefit beyond this intake. } \\
\text { Participants in the control group maintained } \\
>5 \text { serving/week of fish at the end of the trial } \\
\text { (median intake: } 98 \mathrm{~g} / \mathrm{d} \text { ) and the intake of } \\
\text { long-chain omega- } 3 \text { fatty acids was }>700 \\
\mathrm{mg} / \mathrm{d} \text { (median intake: } 703.4 \mathrm{mg} / \mathrm{d} \text { ), well } \\
\text { beyond the threshold of } 500 \mathrm{mg} / \mathrm{d} \text { for } \\
\text { cardiovascular protection. }\end{array}$ \\
\hline $\begin{array}{l}\text { Baseline characteristics. There were } \\
\text { differences in baseline characteristics among } \\
\text { the three intervention groups }\end{array}$ & $\begin{array}{l}\text { - The observed imbalances were minor in size } \\
\text { and clinically irrelevant. } \\
\text { - The only noticeable imbalance from a } \\
\text { clinical point of view was a } 5 \% \text { lower } \\
\text { proportion of women in the MedDiet plus } \\
\text { nuts group. However, this imbalance would } \\
\text { have produced a higher risk of cardiovascular } \\
\text { disease (CVD) in that group, because female } \\
\text { sex is usually associated with reduced risk of } \\
\text { CVD. } \\
\text { - The main results did not materially change } \\
\text { after adjustment for all potential confounding } \\
\text { factors. }\end{array}$ \\
\hline $\begin{array}{l}\text { Drop-outs. There were more dropouts in the } \\
\text { control group and in the MedDiet } \\
\text { supplemented with nuts than in the MedDiet } \\
\text { supplemented with extra-virgin olive oil }\end{array}$ & $\begin{array}{l}\text { - Participants lost to follow-up had a worse } \\
\text { cardiovascular risk profile at baseline. This } \\
\text { would induce a bias towards a benefit for the } \\
\text { control group, hence it does not provide an } \\
\text { alternative explanation to our findings. } \\
\text { - The lower retention rate in the group } \\
\text { randomly allocated to a low-fat diet is a result } \\
\text { in itself. It manifests the inherent difficulty } \\
\text { for participants to be compliant with a low-fat } \\
\text { diet on the long term. }\end{array}$ \\
\hline
\end{tabular}




\begin{tabular}{|c|c|}
\hline \multicolumn{2}{|l|}{ Interpretation of the results } \\
\hline $\begin{array}{l}\text { Specific CVD outcomes. MedDiet only } \\
\text { protected against stroke but not against } \\
\text { myocardial infarction or cardiovascular } \\
\text { mortality }\end{array}$ & $\begin{array}{l}\text { - As is customary in cardiovascular trials, we } \\
\text { tested a composite of cardiovascular events } \\
\text { that was the predefined primary endpoint in } \\
\text { our protocol. The sample size and statistical } \\
\text { power calculations were done assuming this } \\
\text { combined endpoint. } \\
\text { - The confidence intervals for the estimates of } \\
\text { each separate component of the primary } \\
\text { endpoint (stroke, myocardial infarction or } \\
\text { cardiovascular death) showed wide } \\
\text { overlapping and the point estimates for each } \\
\text { of these components for the MedDiet vs. } \\
\text { control always were in the beneficial } \\
\text { direction. } \\
\text { - Low statistical power may explain the non- } \\
\text { significant associations for individual } \\
\text { components. }\end{array}$ \\
\hline $\begin{array}{l}\text { All-cause mortality. PREDIMED did not find } \\
\text { significant results for total mortality, therefore } \\
\text { the MedDiet only changed the causes of death }\end{array}$ & $\begin{array}{l}\text { - Most studies are not powered enough to } \\
\text { show statistically significant results for total } \\
\text { mortality. } \\
\text {-In addition, a larger induction period from } \\
\text { exposure to the reduction in total mortality } \\
\text { needs to be allowed to observe an impact on } \\
\text { all-cause mortality } \\
\text { - The sample size was estimated in order to } \\
\text { obtain differences between groups in the } \\
\text { incidence of the composite endpoint, not total } \\
\text { mortality. To analyze total mortality, we } \\
\text { would need a much larger sample size and a } \\
\text { longer follow-up. }\end{array}$ \\
\hline
\end{tabular}

